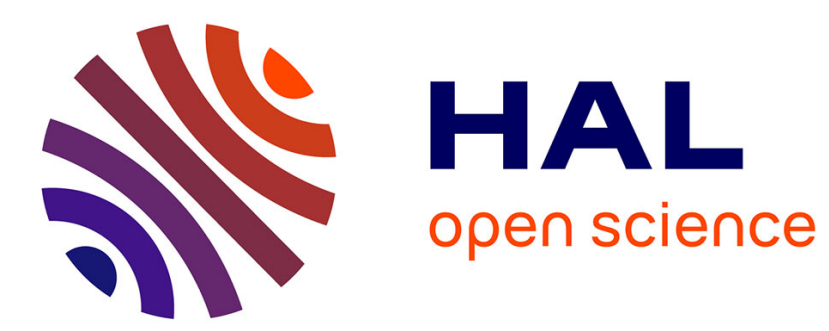

\title{
Activation Parameters of the Zener Peak in Cu-Li Alloys Produced by Electrodeposition
}

\author{
O. Lambri, A. Morón-Alcain, E. Bulejes, A. Penaloza, M. Ortiz
}

\section{To cite this version:}

O. Lambri, A. Morón-Alcain, E. Bulejes, A. Penaloza, M. Ortiz. Activation Parameters of the Zener Peak in Cu-Li Alloys Produced by Electrodeposition. Journal de Physique IV Proceedings, 1996, 06 (C8), pp.C8-89-C8-92. 10.1051/jp4:1996817 . jpa-00254605

\section{HAL Id: jpa-00254605 https://hal.science/jpa-00254605}

Submitted on 1 Jan 1996

HAL is a multi-disciplinary open access archive for the deposit and dissemination of scientific research documents, whether they are published or not. The documents may come from teaching and research institutions in France or abroad, or from public or private research centers.
L'archive ouverte pluridisciplinaire HAL, est destinée au dépôt et à la diffusion de documents scientifiques de niveau recherche, publiés ou non, émanant des établissements d'enseignement et de recherche français ou étrangers, des laboratoires publics ou privés. 


\title{
Activation Parameters of the Zener Peak in Cu-Li Alloys Produced by Electrodeposition
}

\author{
O.A. Lambri*,(1), A.V. Morón-Alcain**, E.D. Bulejes**, A. Peñaloza*** and M. Ortiz*** \\ * Universidad Nacional de Rosario, Departamento de Física, Avda. Pellegrini 250, 2000 Rosario, \\ Argentina \\ ** Laboratorio de Espectroscopía Mecánico Dinámica, Instituto de Física Rosario (IFIR-CONICET-UNR), \\ Avda. 27 de febrero 210 bis, 2000 Rosario, Argentina \\ *** Instituto de Física, Universidad Católica de Valparaíso, Avda. Brasil 2950, Valparaíso, Chile
}

\begin{abstract}
Copper-lithium alloys were produced by electrodeposition. They show a Zener peak at $650 \mathrm{~K}$ (at $0.2 \mathrm{~s}^{-1}$ ) which appears overlapped with the low and intermediate temperature grain boundary damping peaks of copper and also with the phase transition peak related to $\mathrm{Cu}-18$ at $\% \mathrm{Li}$ crystals. In this work the activation energy, $\mathrm{H}$, and the pre-exponential factor $\tau_{0}$ were calculated. Those were $H=(160 \pm 4) \mathrm{KJ} / \mathrm{mol}$ and $\tau_{0}=2 \times 10^{(-14 \pm 1)}(\mathrm{s})$.
\end{abstract}

\section{INTRODUCTION}

Copper lithium alloys exhibit a Zener relaxation peak at $650 \mathrm{~K}$ when the damping is measured about $0.2 \mathrm{~s}^{-}$ 1 This peak appears during the heating and it is absent during cooling owing to the precipitation of lithium from solid solution. The Zener peak appears overlapped with the low and high temperature grain boundary damping peaks of copper, and the phase transition peak of the $\mathrm{Cu}-18$ at $\% \mathrm{Li}$ crystals [1].

In this work the activation parameters of this Zener peak have been calculated through measurements carried out at several, very different, oscillation frequencies [2] and also it has been calculated employing the differential moment of inertia technique $[3,4]$.

\section{EXPERIMENTAL PROCEDURE}

The copper-lithium alloys were obtained by electrodeposition, by using a sheet of high purity copper $(99.99 \%)$ as the cathode and similar size sheet of graphite as the anode. The electrodeposition was made in a bath of fused salts of lithium at constant temperature about $753 \mathrm{~K}$. When the electrodeposition was finished the samples were washed in water for removing the lithium excess [5]. This process allowed to observe that the cathode presented a yellow surface with powders adhered to it with an uneven distribution. These powders are crystals of $\mathrm{Cu}-\mathrm{Li}$ which have an amount of $18 \mathrm{at} \% \mathrm{Li}$, obtained by emission chemical analysis. The external layer of crystals was removed by means of mechanical polishing. The employed sample in the mechanical spectroscopy test had two phases, copper and copper-lithium [1]. Different electrodeposition times were employed which permit to obtain different concentrations of lithium in the sample. They were, $1.07,1.5$ and 2.01 at. \% Li, determined by atomic absorption analysis.

Three types of pendula were used. One is an inverted torsion pendulum at variable moment of inertia which operates at low frequency from $0.2 \mathrm{~Hz}$ up to $40 \mathrm{~Hz}$ [3]. Other one is also, an inverted torsion pendulum at variable moment of inertia, but operated at medium frequency from $30 \mathrm{~Hz}$ up to $150 \mathrm{~Hz}$ [3]. Another one is an inverted micropendulum working in torsion, which has very small moment of inertia [4] like the employed ones for measuring metallic glasses ribbons. The three pendula work at low and high temperatures under high vacuum and are driven by a PCL-812 Card The usual optical system, including a mirror and a photodiode, was employed for recording the oscillations which were analysed by means of the data acquisition system [3].

The samples were in sheets of $2 \mathrm{~mm}$ wide, $0.1 \mathrm{~mm}$ thick and $20 \mathrm{~mm}$ long. and the maximum shear strain, $\varepsilon$, was less than $5 \times 10^{-5}$. Heating was made up to $1010 \mathrm{~K}$ with an increasing rate of $1 \mathrm{~K}$ per minute.

(1) Present address: Departamento de Física de la Materia Condensada, Facultad de Ciencias, Universidad del País Vasco, Apdo. 644, 48080 Bilbao, Spain 
Decreasing temperature was also made at the same rate. The damping values were measured in free decay with an error less than $1 \%$ and were considered amplitude independent. In fact, the corrections made to the measured damping for obtaining the intrinsic values result non greater than $5 \times 10^{-5}$. Therefore, the amplitude dependent damping effects are within the experimental error range and can be neglected without obstructing the subsequent calculations made in this work.

The damping background behaviour as a function of temperature was determined by means of the damping response of a completely liquid rheological model. In other words, the dynamical response of the Voigt standard anelastic solid rheological model with the spring unrelaxed member changed by a dashpot has been considered. In fact, the behaviour of the three rheological parameters as a function of temperature is more smooth than the damping background exponential law [1].

The activation parameters were calculated by means of two procedures with the aim of obtaining accurate values, even if this Zener peak does not appear clear.. The first method was to calculate the preexponential factor, $\tau_{0}$, and the activation energy, $\mathrm{H}$, of the relaxation time, $\tau$, which satisfies an Arrhenius law, by means of the ordinate and the slope of the well kwon plot $\ln \left(\omega^{-1}\right)$ against $1 / T$ [2]. Where $\omega=2 \pi f, f$ being the frequency of the applied stress and $T$ the absolute temperature. The second method was employing the moment of inertia variable technique [3,4]. In fact, through the partial derivative with respect to the moment of inertia ( I ), of the general expression for the internal friction (F), the $\ln \tau$ can be calculated as a function of temperature [4], that is

$$
\ln \tau \equiv \operatorname{arght}\left(\left(-\frac{\partial \ln F}{\partial \ln \omega}\right)_{(1 / \mathrm{T})}\right)-\ln \omega
$$

The goal of this procedure is the possibility of determining the activation parameters with only one peak, without need other peak at higher frequencies and also the possibility of obtaining several values of $\ln (\tau)$ as a function of temperature in an unique peak, wherever the Arrhenius behaviour does not deviate and the argth can be calculated [4]. The calculus of the partial derivative was made as follows : at each temperature $F$ is measured at a fixed moment of inertia (in this case in free decay), leading to $F_{1}$ and $\omega_{1}$, subsequently the moment of inertia is changed slightly giving new values to $F$ and $\omega$, namely $F_{2}$ and $\omega_{2}$. This way of measuring allows, the calculation of the partial derivatives of $F$ and $\omega$ with respect to the moment of inertia at constant temperature $[3,4]$.

\section{RESULTS AND DISCUSSION}

Figure 1 shows the damping spectra measured during heating at approximately $0.2 \mathrm{~s}^{-1}$ for the Cu-Li alloys with three different lithium content. In figure 2 are shown the mean peaks obtained from the spectra of figure 1 after the background subtraction. These peaks are produced owing to the contribution of four effects, the low and intermediate temperature grain boundary peaks of copper (called LTP and ITP), the phase transition peak of the crystals of $\mathrm{Cu}-18$ at.\% Li (PTP) and the Zener peak (ZP) [1]. During the cooling the ZP does not appear due to the precipitation of lithium in solid solution. Furthermore the PTP neither appears because of the fact that the melting of the crystals has occurred during the heating. Only were measured during the cooling the LTP and ITP peaks [1].

Deconvolution of the measured spectra was made by means of Peak Fit 4.0 soft through the second derivative study. In fact, this mode of deconvoluting the spectrum was chosen, but it is not unique [8]. The peak temperatures of the LTP, ITP and PTP were given as a guess value and the shape and the peak temperature of the $Z P$ was deconvoluted. The peak temperature of the LTP and the ITP was previously determined considering the mean value of both the peak temperature in the unelectrodeposited samples and in the electrodeposited ones. In the electrodeposited samples the behaviour during the heating and cooling was also considered. The peak temperature of them during heating and cooling in the electrodeposited samples changed slowly and it did not change in the unelectrodeposited ones [1]. In the determination of the peak temperature of the PTP the change in shape of the damping spectrum when the frequency is increased was considered. This change allows to separate considerably the PTP of the ZP [1]. The 
activation energy for the LTP and ITP, obtained when the frequency is changed towards higher values resulted in agreement with the reported values in the literature [9]. Moreover the width at half maximum of the deconvoluted LTP and ITP resulted also in agreement with the reported values. The deconvoluted peaks are plotted in figure 2 by means of full lines.

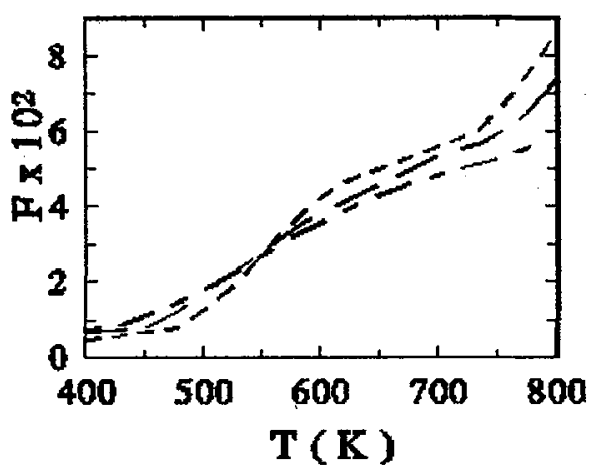

Figure.:1 : Damping spectra for the $\mathrm{Cu}-\mathrm{Li}$ alloys. Alt dashed line 1.07 at \% $\mathrm{Li}$, long dashed line 1.5 at $\% \mathrm{Li}$ and short dashed line 2.01 at $\% \mathrm{Li}$.
The peak height of both, the total peak and the deconvoluted $Z \mathbf{P}$, varied in a linear mode as a function of square lithium concentration. The peak height, taken as a relative measure of the relaxation strength, as a function of square concentration is plotted in figure 3 where a linear behaviour can be seen, which is in agreement with the Zener's pair reorientation theory [2]. This result must be considered with care, because of the fact that the ZP is overlapped with the LTP, the PTP and the ITP. The LTP and ITP peaks characteristics could change in different degrees during the run up in temperature for samples with different electrodeposition times, even if they are similar during the run down in temperature.

The PTP peak characteristics also could change for samples with different electrodeposition times and it will be discussed elsewhere. Furthermore, another point must be considered, it is the variation of the lithium in solid solution during the run up in temperature [1].

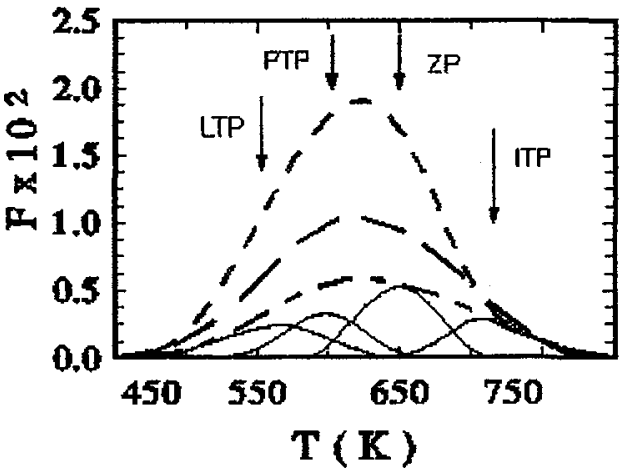

Figure 2: Mean peaks in the $\mathrm{Cu}-\mathrm{Li}$ alloys measured during heating. Alt dashed $1.07 \mathrm{at} \% \mathrm{Li}$, long dashed $1.5 \mathrm{at} \% \mathrm{Li}$, short dashed 2.01 at\% Li.

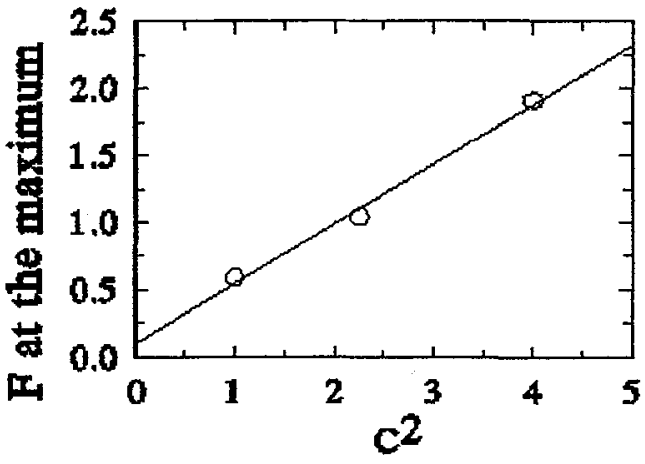

Figure 3: $\mathrm{F}$ at the maximum height against the atomic lithium fraction, $c$, for the peaks of figure 1 .

It should be mentioned that in a next work [10] performed in electrodeposited single crystals, the assumption of the arising of the ZP made in the early results of Ref. [1] is widely corroborated. In fact, one of the studied points is the splitting of the PTP from the ZP against the change in frequency and the dependence of the ZP peak height with the square lithium concentration.

In the first procedure for obtaining the activation parameters, the indetermination in the frequency values owing to the overlapping of the processes above mentioned is other error added to the indetermination of the peak temperature. In the second procedure appears the critical problem which is the calculus of the derivative of eq.(1). Even if in this procedure all the peak can be processed, only in the temperature range around the peak temperature, solutions within an Arrhenius law can be obtained owing to the indeterminations produced by the deconvolution process. The Arrhenius plot for the Zener peak in the $\mathrm{Cu}-\mathrm{Li}$ alloys calculated from both method is plotted in figure 4 . The full symbols are related to values calculated by means of the first procedure and the empty ones are related to the second procedure. Each 
set of values was fitted independently of each others. Subsequently, the mean value, with the same weight, of $\mathrm{H}$ and $\tau_{0}$ was calculated. Even if the evaluated range is small in the values calculated through the differential moment of inertia technique, the correlation is higher than in the values obtained commonly. The obtained values were $\mathrm{H}=(160 \pm 4) \mathrm{KJ} / \mathrm{mol}$ and $\tau_{0}=2 \times 10^{(-14 \pm 1)}(\mathrm{s})$.

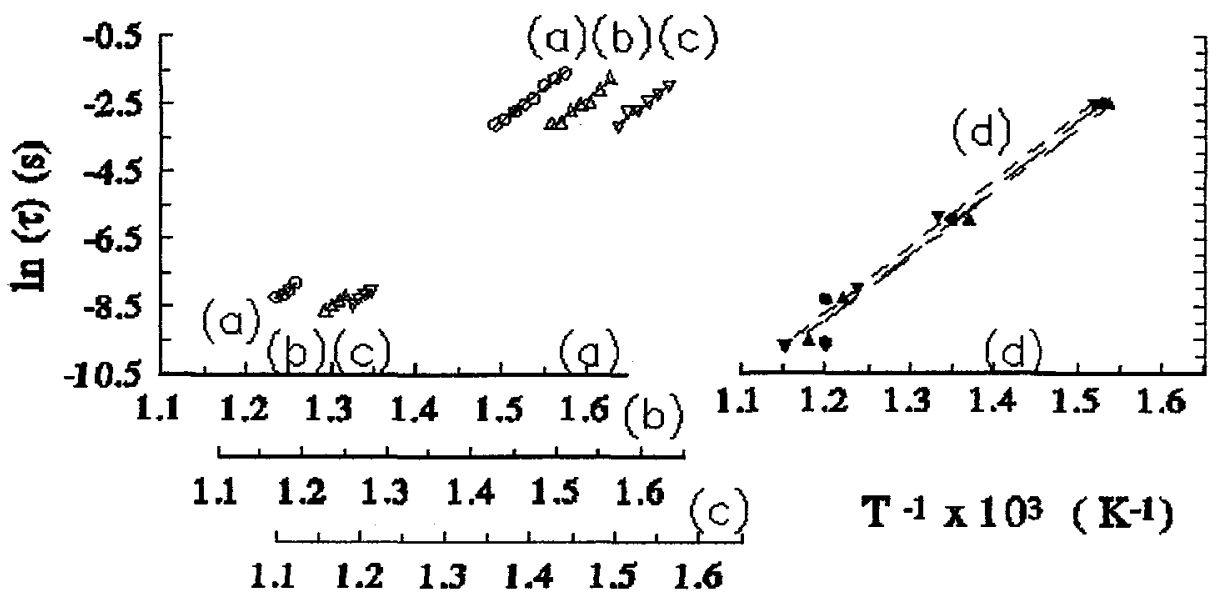

Figure 4: Arrhenius plot for the Zener peak in Cu-Li alloys. The labels indicate the reference $\mathrm{x}$-axis for each curve. Circle 1.07 at $\% \mathrm{Li}$. triangle 1.5 at. $\% \mathrm{Li}$, inverted triangle 2.01 at. $\% \mathrm{Li}$.

\section{CONCLUSION}

The activation parameters in the Zener relaxation which occurs in copper-lithium alloys were calculated. Those are activation energy $(160 \pm 4) \mathrm{KJ} / \mathrm{mol}$ and pre-exponential factor $\left.2 \times 10^{(-14} \pm 1\right)(\mathrm{s})$.

\section{Acknowledgements}

This work was supported partially by the CONICET, the Antorchas Foundation and the PMMA / OAS CNEA. O.A. L whishes to express his appreciation to CONICET by an external fellowship.

\section{References}

[1] Lambri O. A., Peñaloza A., Morón-Alcain A. V., Ortiz M. and Lucca F. C., Mater Sci \& Eng. A., 212 (1996), 108.

[2] Nowick A. S. and Berry B. S., Anelastic relaxation in crystalline solids (Acad. Press, New York, 1972).

[3] Lambri O. A, Phd. Thesis Rosario National University, Rosario, Argentina (1993).

[4] Lambri O. A., Mater. Trans., JM, 35, 7 (1994), 458.

[5] Peñaloza A., Ortiz M. and Worner C., J. of Mat. Sci. letters, 14 (1995), 511.

[6] Bulejes E. D., Sánchez F. A., Lambri O. A. and Povolo F, Report, Rosario Institute of Physics, (Rosario, Argentina 1994), 02/94.

[7] Peak Fit, Jandel Scientific Software, AISN (1995).

[8] Ritchie I. G., Personal communication

[9] Ashmarin G. M., Golubev M. Y., Naumova N. Y. and Shalimova A. V., Internal friction and ultrasonic attenuation in solids, International Academic Pulishers, Beijing China, (1990), 385.

[10] Lambri O. A., Peñaloza A., Morón-Alcain A. V., Ortiz M. and Lucca F. C, to be published. 